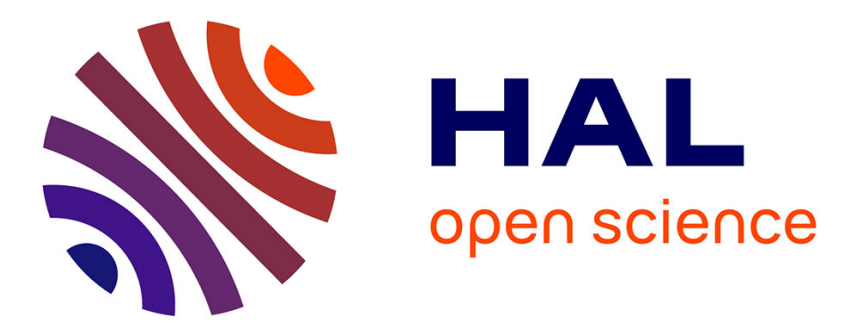

\title{
Ionizing Doses Calculations for Low Energy Electrons in Silicon and Aluminum
}

\author{
Juliette Pierron, Christophe Inguimbert, Mohamed Belhaj, Melanie Raine, \\ Jérôme Puech
}

\section{> To cite this version:}

Juliette Pierron, Christophe Inguimbert, Mohamed Belhaj, Melanie Raine, Jérôme Puech. Ionizing Doses Calculations for Low Energy Electrons in Silicon and Aluminum. IEEE Transactions on Nuclear Science, 2017, 64 (8), p. 2340-2348. 10.1109/TNS.2017.2662220 . hal-01629210

\section{HAL Id: hal-01629210 https://hal.science/hal-01629210}

Submitted on 6 Nov 2017

HAL is a multi-disciplinary open access archive for the deposit and dissemination of scientific research documents, whether they are published or not. The documents may come from teaching and research institutions in France or abroad, or from public or private research centers.
L'archive ouverte pluridisciplinaire HAL, est destinée au dépôt et à la diffusion de documents scientifiques de niveau recherche, publiés ou non, émanant des établissements d'enseignement et de recherche français ou étrangers, des laboratoires publics ou privés. 


\title{
Ionizing Dose Calculations for Low Energy Electrons in Silicon and Aluminum
}

\author{
J. Pierron $^{1}$, C. Inguimbert ${ }^{1}$, M. Belhaj ${ }^{1}$, M. Raine ${ }^{2}$, and J. Puech ${ }^{3}$
}

1) ONERA-DESP, 2 av. E. Belin, 31055 Toulouse, France

2) CEA, DAM, DIF, F-91297 Arpajon, France

3) French Space Agency CNES, 18 av. E. Belin, 31401 Toulouse cedex, France.

J. Pierron is with ONERA-DESP, 2 av. E. Belin, 31055 Toulouse, France (tel: 33-562252405, email: Juliette.Pierron@ onera.fr).

C. Inguimbert is with ONERA-DESP, 2 av. E. Belin, 31055 Toulouse, France

(tel: 33-562252734, email: Christophe.Inguimbert@ onera.fr).

M. Belhaj is with ONERA-DESP, 2 av. E. Belin, 31055 Toulouse, France (tel: 33-562252937, email: Mohamed.Belhaj@ onera.fr).

M. Raine is with CEA, DAM, DIF, F-91297 Arpajon, France

(email: Melanie.Raine@cea.fr).

J. Puech is with CNES-DCT/RF/HT, 18 av. E. Belin, 31401 Toulouse, France (tel: 33-561273510, email: Jerome.Puech@cnes.fr ).

\begin{abstract}
The electron transport at low energy (10 eV - $2 \mathrm{keV})$ is investigated with a Monte Carlo code. Ionizing doses are presented down to $\mathbf{1 0} \mathrm{eV}$ for aluminum and silicon, and correlated with electron emission yields.
\end{abstract}

Index terms- Ionizing dose, low energy electrons, Monte Carlo code.

Paper submitted to RADECS 2016 TNS Special Issue

No. TNS-00532-2016.R3 


\title{
Ionizing Dose Calculations for Low Energy Electrons in Silicon and Aluminum
}

\author{
J. Pierron, C. Inguimbert, M. Belhaj, M. Raine, and J. Puech
}

\begin{abstract}
The electron transport at low and very low energy $(10 \mathrm{eV}-2 \mathrm{keV})$ is investigated with a Monte Carlo code in silicon and aluminum. The elastic scattering with nuclei is described by Mott's model of partial waves, whereas the inelastic collisions with electrons are described by the complex dielectric function theory. Comparisons of Monte Carlo simulations with electron emission yields (EEY) and energy loss spectra experimentally measured in ultrahigh vacuum on Ar-etched samples are given. The practical ranges and the ionizing dose calculations are presented down to $10 \mathrm{eV}$ for electrons in silicon and aluminum. The simulation results show a correlation between the EEY and the ionizing doses. At low energy, while the electrons stay in the first $\sim 10 \mathrm{~nm}$ from the surface due to the elastic scattering, the EEY increases and the ratio of the ionizing dose over the incident energy decreases. Above $200 \mathrm{eV}$, when the electrons go deeper into the solid due to the inelastic scattering, the EEY decreases and the ionizing dose ratio increases.
\end{abstract}

Index terms - ionizing doses, low energy electrons, Monte Carlo code.

\section{INTRODUCTION}

$\mathrm{T}$ The study of low energy electron transport in matter is of great interest for many fields of applications. For instance, the amount of electrons emitted by a surface when hit by an incident electron, or Electron Emission Yield (EEY), is of particular importance for particle accelerators and satellite communication devices performances [1]-[2]. In the latter, electrons of a few tens of $\mathrm{eV}$ are known to cause vacuum discharges (Multipactor effect) that can degrade the devices or, at least, limit the transmitted power. In microwave and millimeter wave power tubes, if the EEY is larger than a value of 1 , undesirable electron-clouds can be produced. These electron-clouds can lead to the triggering of electrostatic discharges that disturb the transmitted signal. This phenomenon can be mitigated by using low secondary-electron-emission materials. Similarly, the penetration depth of incident electrons is an important parameter for surface spectroscopy analysis [3] and in microelectronic devices submitted to the radiations of the space environment. The reduction in size of electronic devices which has recently reached about $14 \mathrm{~nm} \mathrm{[4]}$ requires the use of more and more accurate radiation transport codes. For instance, advanced electronics devices of small dimensions are demonstrated to be sensitive to single event

This paper was submitted to RADECS 2016 TNS Special Issue in September 2016. J. Pierron, C. Inguimbert, and M. Belhaj are with ONERA, 2 av. E. Belin, 31055 Toulouse, France (tel: 33-562252734, email:

Juliette.Pierron@onera.fr, Christophe.Inguimbert@onera.fr,

Mohamed.Belhaj@onera.fr). M. Raine is with CEA, DAM, DIF, F-91297 Arpajon, France (email: Melanie.Raine@cea.fr ). J. Puech is with CNES,

18 av. E. Belin, 31401 Toulouse, France (email: Jerome.Puech@cnes.fr ). effects induced by secondary electrons created along the track of heavy ions and protons [5]. Some devices with a technological node of $\sim 16 \mathrm{~nm}$ having a sensitive volume of some tens of nanometers and a critical charge of some tenths of $\mathrm{fC}$ are demonstrated to be sensitive to incident electrons [5]-[6]. At these scales, the radiation transport modeling shall be accurate over small distances of few nanometers and very low energies of few tens of eV. Hence, the prediction of risk of single event effects requires some accurate electron transport codes valid down to a few eV at scales of some nanometers. Such kind of transport necessitates the development of specific models based on an accurate description of physics. Some authors have worked in the past to develop such kind of electron transport codes (references [7]-[14] and the references therein). Recently, a new module dedicated to low energy electron transport has been implemented in Geant4 [15]. This electromagnetic model of Geant 4 called MicroElec performs the electron transport down to $\sim 16 \mathrm{eV}$ in silicon [9]-[11]. It has been used to improve the modeling of heavy ion track structures below a radial distance of $10 \mathrm{~nm}$ [10]. The Geant4MicroElec library extension [9]-[11] has also been employed to investigate the sensitivity of advanced devices to incident electrons [5]-[6].

For now, the Geant4-MicroElec model is only valid down to $\sim 16 \mathrm{eV}$ for a single material (silicon). Some other codes have been implemented over the past years for silicon [8], [12]-[14]. ONERA in collaboration with CNES has developed its own code: OSMOSEE [16]-[17]. In this code dedicated to low energy electron emission, the implemented physical models were initially specific to aluminum. On the other side, the Geant4-MicroElec library extension is based on the complex dielectric function theory and could be easily adapted to different materials. In a collaborative effort, this work proposes to extend the domain of validity of such kind of approaches to different materials and down to a few eV. At these low energies additional phenomena (not included in MicroElec), for example surface plasmon excitations or phonons, must be taken into account. Up to now, three materials (aluminum, silver, and silicon) have been implemented in the code OSMOSEE for energies between $10 \mathrm{eV}$ and $2 \mathrm{keV}$ [18]. This enables the estimation of some relevant parameters, such as the ionizing dose and the range of electrons, useful for new generation devices of small dimensions.

In this paper, simulations from the Monte Carlo (MC) code OSMOSEE are compared with experimental measurements of Electron Emission Yields (EEY) and energy loss spectra obtained in ultrahigh vacuum on Ar-etched samples with a facility entirely dedicated and designed to electron emission [19][20]. 


\section{LOW ENERGY MONTE CARLO CODE}

In matter, the trajectory of electrons is driven by two main mechanisms: the elastic and the inelastic scatterings. In the code OSMOSEE, the elastic interactions (i.e. deflection by nuclei) are described by Mott's model (or partial waves) from the code ELSEPA [21]. The inelastic scattering (i.e. collisions with electrons) is described by the complex dielectric function theory [9], [13], [22] and optical data [23]. The code takes into account surface plasmon excitations, phonons, and the crossing of the surface potential barrier [16]-[17]. The MC procedure is a standard one. Random numbers are used to determine the type of interaction, the distance traveled between collisions, and the characteristics of the interaction (scattering angles, energy transfers). It has been described in previous papers [16]-[17]. Paths and energy losses are calculated until the electron is emitted out of the solid or until its energy falls under an energy threshold. This energy cutoff equals e $\chi+E_{G}+\Delta V$ for semiconductors and $E_{F}+W$ for metals, with $E_{G}$ the energy bandgap, e $\chi$ the electron affinity, $\Delta V$ the valence bandwidth, $\mathrm{E}_{\mathrm{F}}$ the Fermi level and $\mathrm{W}$ the work function.

In section III, the principle of the simulation method (complex dielectric function theory) is briefly summarized. A comparison with experimental measurements is presented in section IV. Finally, the practical ranges, and the ionizing dose profiles are presented for silicon and aluminum targets in section $\mathrm{V}$.

\section{INELASTIC MEAN FREE PATHS}

In the complex dielectric function theory, the total inelastic mean free path $\lambda$ can be calculated using equation (1) where $\mathrm{a}_{0}=5.29 \times 10^{-11} \mathrm{~m}$ is the Bohr radius, $T$ the kinetic energy of the incident electron, and $\varepsilon(\hbar \omega, \hbar q)$ the exact dielectric function of the solid with $\hbar \omega$ and $\hbar q$ the energy and momentum transferred to the electrons of the solid [22].

$$
\frac{d^{2} \lambda^{-1}}{d \hbar \omega d \hbar q}=\frac{1}{\pi a_{0} T} \operatorname{Im}\left[\frac{-1}{\varepsilon(\hbar \omega, \hbar q)}\right] \frac{1}{\hbar q}
$$

The first step of this method is to model the imaginary part of the inverse of the dielectric function (energy loss function) that contains all information on the electronic band structure of the solid. The energy loss function for $\hbar q=0$ may be obtained from optical data [22]-[23] and modeled with a sum of Drude type functions (2) with $A(k), \Gamma(k, 0)$, and $E(k, 0)$, respectively the height, the width, and the energy of the kpeak on the energy loss function at $\mathrm{q}=0$ [9]. The function $\Theta$ is a Heaviside function used to model the energy threshold $E_{b k}(k)$ of the k-shell.

$$
\begin{aligned}
& \operatorname{Im}\left[\frac{-1}{\varepsilon(\hbar \omega, \hbar q=0)}\right] \\
& =\sum_{k} \frac{A(k) \Gamma(k, 0) \hbar \omega \times \Theta\left(\hbar \omega-E_{b k}(k)\right)}{\left(E(k, 0)^{2}-(\hbar \omega)^{2}\right)^{2}+(\hbar \omega)^{2} \Gamma(k, 0)^{2}}
\end{aligned}
$$

The methods to extend this function to all momentums $\hbar q$ have been described by different authors [7], [9], [13], [22], [24]. Following Kuhr and Fitting [13] we used two dispersion relations (3) and (4) to model the energy shift and the plasmon damping with $E_{p l}$ the volume plasmon energy in the free electron gas theory, $\mathrm{m}$ the electron mass, $\mathrm{Z}$ the atomic number and $\beta$ a constant.

$$
\begin{aligned}
& E(k, q)=E(k, 0)+\frac{6 E_{F}(\hbar q)^{2}}{5 E_{p l} \times 2 m} \\
& \Gamma(k, q)=\Gamma(k, 0)\left(1+\beta q^{2}\right)
\end{aligned}
$$

Consistency of the fitting [7], [9], [22] is given by three sum-rules (5), (6) and (7), with $I$ the mean excitation potential. The maximum discrepancy was of less than $8 \%$ for silicon and of less than $12 \%$ for aluminum.

$$
\begin{gathered}
\frac{2}{\pi E_{p l}{ }^{2}} \int_{0}^{\infty} \operatorname{Im}\left[\frac{-1}{\varepsilon(\hbar \omega, \hbar q=0)}\right] \hbar \omega d(\hbar \omega)=Z \\
\frac{2}{\pi} \int_{0}^{\infty} \operatorname{Im}\left[\frac{-1}{\varepsilon(\hbar \omega, \hbar q=0)}\right] \frac{d(\hbar \omega)}{\hbar \omega}=1 \\
\ln (I)=\frac{2 \int_{0}^{\infty} \operatorname{Im}\left[\frac{-1}{\varepsilon(\hbar \omega, \hbar q=0)}\right] \hbar \omega \ln (\hbar \omega) d(\hbar \omega)}{Z \pi E_{p l}{ }^{2}}
\end{gathered}
$$

To get the total inelastic mean free path $\lambda$, one has to integrate equation (1) over energy $\hbar \omega$ and momentum $\hbar q$ transfers. The boundaries in $\hbar q$ are determined [7], [9], [24] by the law of conservation of energy and momentum (8) given for non-relativistic electrons.

$$
q_{\mp}(T, \hbar \omega)=\frac{\sqrt{2 m}}{\hbar}[\sqrt{T} \mp \sqrt{T-\hbar \omega}]
$$

The boundaries in energy transfer $\hbar \omega$ are obtained with respect to the Pauli principle [7], [9], [13], [24]. In the MC code OSMOSEE, the bottom of the valence band has been chosen as an energy reference for electrons in semiconductors, and the bottom of the conduction band as an energy reference for electrons in metals. Equation (9) provides the total inelastic mean free path $\lambda$ for electrons in silicon [25], $E$ being the total energy of the electron in the solid. For aluminum, $E_{G}+\Delta V$ is replaced by $E_{F}$. The total inelastic mean free path $\lambda$ calculated with (9) is shown on Fig. 1 for silicon.

$$
\begin{aligned}
& \lambda^{-1}(E)=\frac{1}{\pi a_{0}\left(E-E_{G}-\Delta V\right)} \\
& \times \int_{E_{G}}^{E-E_{G}-\Delta V} \int_{q_{-}\left(E-E_{G}, \hbar \omega\right)}^{q_{+}\left(E-E_{G}, \hbar \omega\right)} \operatorname{Im}\left[\frac{-1}{\varepsilon(\hbar \omega, \hbar q)}\right] \frac{1}{\hbar q} d(\hbar q) d(\hbar \omega)
\end{aligned}
$$

At high energy, the total inelastic mean free paths available in the literature are in good agreement with one another, however below $50 \mathrm{eV}$, the scatter within the data is significant. The colored area on Fig. 1 shows an image of the dispersion of the 
data from the literature [8]-[9], [12], [14], [26]-[27]. Within the dielectric function theory, uncertainties may arise from the fitting of the optical data but also from the dispersion relations that were chosen. Moreover, the dielectric function theory is based on the First Born approximation that may fail at low energy because of exchange effects in electron-electron interactions [9]. We did not consider corrections for exchange effects in this work. An uncertainty on the definition of the mean free paths of electrons can have a significant impact on the final results. Above $100 \mathrm{eV}$, the discrepancies between the values coming from different simulations [8]-[9], [12], [26] are lower than $12 \%$. It leads to transport calculations in relatively good agreement with one another. This is discussed further in the paper (section V). Below $100 \mathrm{eV}$, the uncertainty is far larger. The dispersion of the data is symbolized by the colored region in Fig. 1. Our calculation is relatively close to the experimental data provided in the references [9], [26]. The discrepancy with the mean free path calculated by Fitting [12] is in the order of $28 \%$ at $10 \mathrm{eV}$. The discrepancy with Shinotsuka's data [26] is similar (21\%). Above $100 \mathrm{eV}$, these discrepancies are respectively of about $4.7 \%$ and $12 \%$ with these two models.

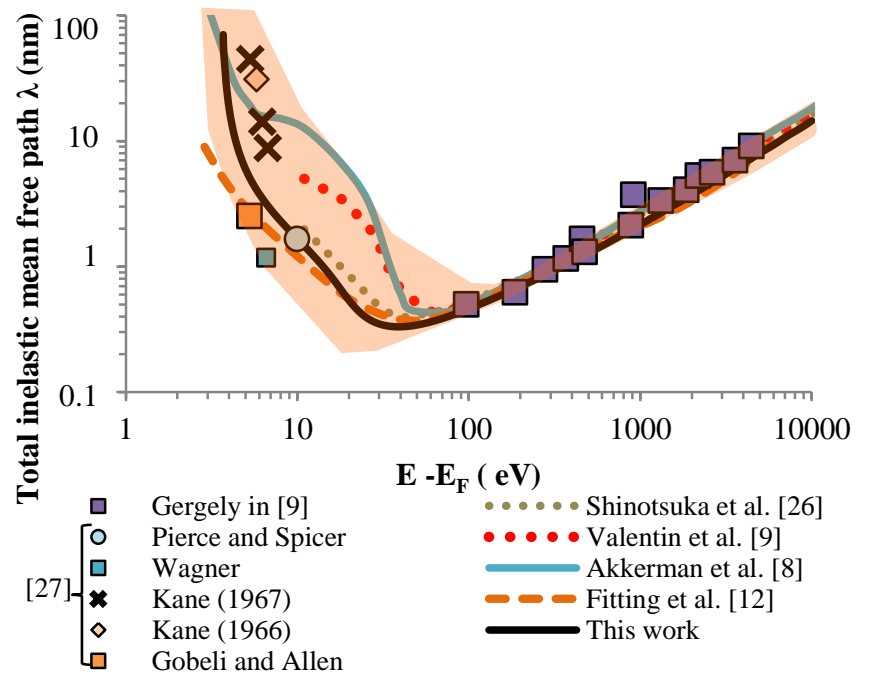

Fig. 1. Total inelastic mean free path for electrons in silicon (after [25]). Full [8], dashed [12] and dotted [9], [26] lines represent calculations. Dots refer to experimental data from the references therein [9], [27], and crosses are used for calculations from a reference therein [27]. The colored area is an image of the dispersion of the data from the literature ([8]-[9], [12], [14], [26]-[27] and the references therein).

To get the partial mean free path of the $\mathrm{i}$-th shell ionization, the boundaries for the energy transfer are $E_{b i}$ and $\left(E+E_{b i}\right) / 2$ with $E_{b i}$ the binding energy for the i-th shell. The maximum energy transfer $\left(E+E_{b i}\right) / 2$ takes into account the exchange effects between the primary and the secondary electrons. The inverse partial mean free paths $\lambda^{-1}$ calculated for each shell are reported in Fig. 2 for silicon. One finds again some discrepancies between the different models. These discrepancies are of the order of $30 \%$ in average over the whole energy range.

A fairly good agreement is found with Gryzinski's model [28], and with the recent calculations of Bote and Salvat [29] which take into account the deformed wave function for elec- trons in the first Born approximation. The first Born approximation used in the MC code assumes that the initial and final states of the incident electron are planes waves. This approximation is demonstrated to be adequate to describe inelastic collisions for small binding effects [30]. However it is less relevant for incident electrons having an energy close to a ionization threshold where the distortion of the incident electron wave function can be important [29]-[30]. Nevertheless, the mean free paths calculated from (9) are shown to be reliable on the whole energy range for Monte Carlo simulations (Fig. 2). At energies higher than $10 \mathrm{keV}$, it is necessary to take into account relativistic corrections into equation (8).

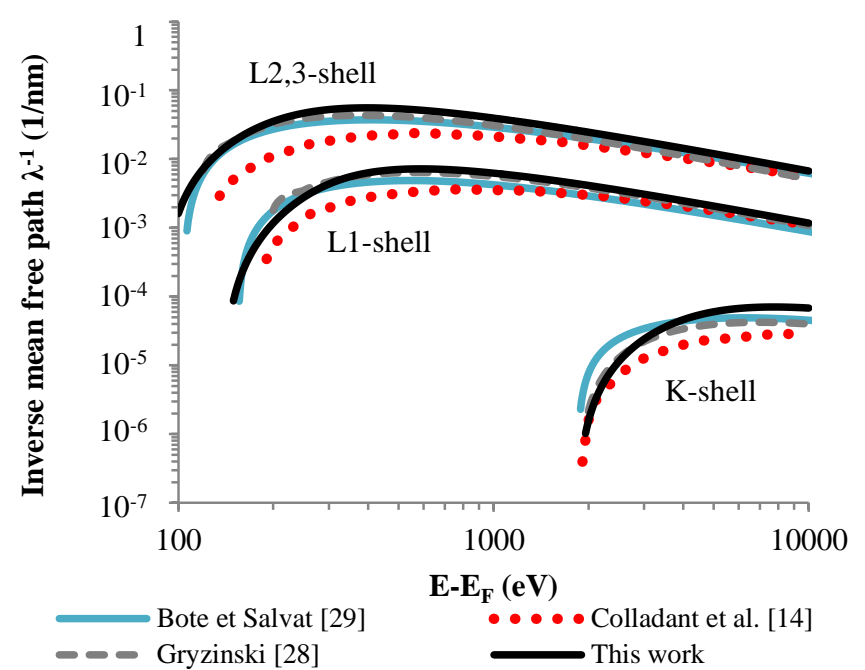

Fig. 2. Contributions for the core electrons to the total inelastic mean free path for silicon. The data come from [14], calculations with Gryzinski's formula [28] and the code of Bote and Salvat and coworkers [29] freely available on the NIST website.

The previous calculations describe the interactions of electrons in the bulk solid. To study the electron transport at low energy, additional phenomena such as surface plasmon excitations and phonons must be considered. The surface plasmon excitations have been modeled introducing in the energy loss function the dispersion relation for plasmons near the surface: $\varepsilon(\hbar \omega, \hbar q)+1=0$ [24]. As a first approximation, the energy loss function to use in the previous equations is then $\operatorname{Im}\left[\frac{-1}{\varepsilon(\hbar \omega, \hbar q)+1}\right]$. As surface plasmons may only occur in the first few nanometers from the surface (i.e. Bregrenzung effect), following [16]-[17], [31] a dependence of the electron depth has been added to the surface plasmon mean free path [25]. Phonons energy losses are simply taken into account assuming only the emission of an optical phonon [32]. It is based on the mean free paths recently calculated by Akkerman et al. [32]. The energy loss is taken equal to $0.05 \mathrm{eV}$ for silicon [33]. No production of secondary electrons is considered during this interaction.

\section{EEY AND ENERGY LOSS SPECTRA}

The transport of low energy electron is of great importance for some applications subject to the multipactor effect. The risk of multipactor discharge is driven by the electron emission 
yield (EEY). This quantity has been extensively studied and a lot of data exist in the literature for comparisons with our simulations. However, there are few data below $1 \mathrm{keV}$ [34][35] and even less for samples with a fairly controlled surface state. The EEY is closely related to the mean free paths of the incident electrons and is a good candidate to check the consistency of the model. In the following, the code OSMOSEE is compared with experimental measurements of EEY and energy loss spectra. All measurements are performed in the ultrahigh $\left(10^{-9}-10^{-10} \mathrm{mbar}\right)$ vacuum facility located at ONERA. A full description of this facility, entirely dedicated and designed for the study of electron emission can be found in references [19]-[20]. It uses an ELG-2 electron gun from Kimball Physics and an ion gun from Tectra for Ar-etching. The electron beam is pulsed during EEY measurements to limit the surface conditioning effect and is continuous during spectra acquisition. During Ar-etching, the ion gun energy is of $1 \mathrm{keV}$ and the angle of incidence is normal to the surface. The facility uses an Omicron hemispherical electron analyzer that can record spectra from 2 to $2000 \mathrm{eV}$. Before being mounted in the measurement system, all samples are washed with ethanol. Prior to measurements, Auger electron spectroscopy is used to monitor the surface composition and cleanliness. During EEY measurements, the sample is negatively biased to $-9 \mathrm{~V}$.

The EEY is defined as the ratio between the number of electrons emitted by a solid over the number of incident electrons. Fig. 3 shows the total EEY for silicon (Fig. 3a) and aluminum (Fig. 3b) targets.

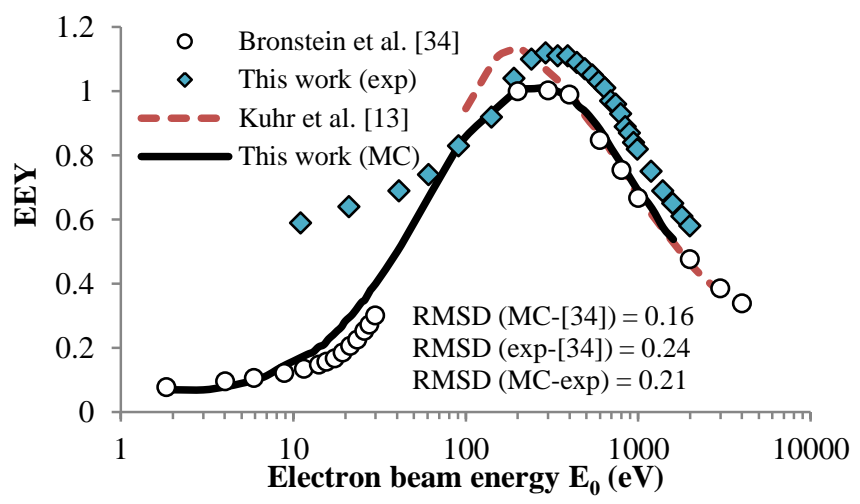

(a)

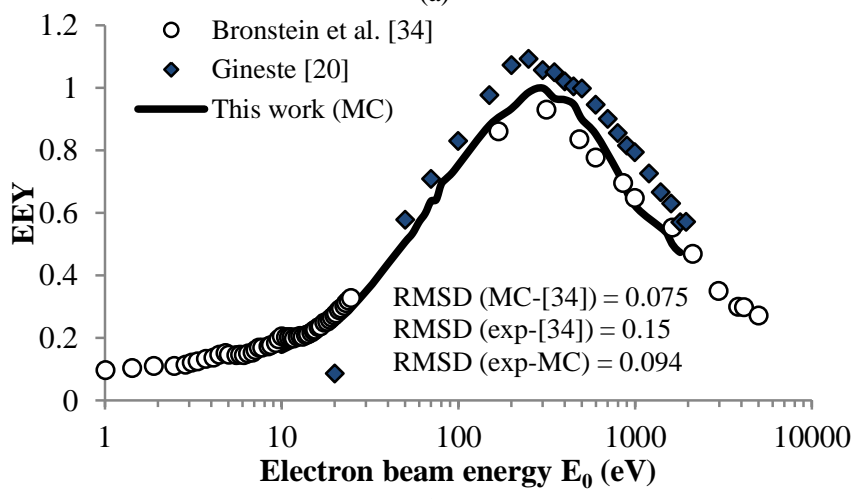

(b)

Fig.3. Total EEY for (a) silicon (after [25]) and (b) aluminum. Full and dashed lines are MC simulations [13]. Dots are experimental measurements from [34] and ONERA (including [20]). Except for the first points, both the experimental $(<10 \%)$ and the numerical $(\sim 3 \%)$ uncertainties are smaller than the size of the symbols used to plot the data.
MC simulations from the code OSMOSEE are found to be in relatively good agreement with our experimental measurements. The mean discrepancy is found to be of $19.3 \%$ for silicon (13\% without the first three points) and of $18.5 \%$ for aluminum (10.3\% without the first point). For silicon, the uncertainty of the experimental EEY is of $0.06(10 \%)$ at $11 \mathrm{eV}$, and then falls below $0.05(8 \%)$ for energies higher than $21 \mathrm{eV}$, and below $0.03(4 \%)$ for energies higher than $191 \mathrm{eV}$. The uncertainties are of the same order of magnitude for aluminum. The simulated EEY has been estimated by the Monte Carlo method with a counting rate of around $\sim 1000$ electrons that represents an error of around $3 \%$. Our results are also in relatively good agreement with Bronstein and Fraiman's experimental data [34] on vacuum evaporated samples and with the MC simulations of Kuhr and Fitting [13]. The Root Mean Square Deviation (RMSD) values are given on Fig. 3. The mean deviation between our experimental data and Bronstein and Fraiman's data is found to be of 0.24 for silicon $(16.5 \%$ without the first points) and of 0.15 for aluminum $(17.5 \%$ without the first point). Similarly, the mean deviation between our MC simulations and Bronstein and Fraiman's data is of 0.16 for silicon (10.3\%) and of 0.075 for aluminum $(9.2 \%)$. For both silicon and aluminum, our MC simulations remain within the experimental uncertainties. However, our MC simulations are closer to Bronstein and Fraiman's data than our experimental measurements. Since our measurements are performed on bulk solids, for easily oxidized materials such as silicon and aluminum, a capping layer of oxide may remain on the sample after Ar-etching. This may explain the discrepancy at very low energy between our data and Bronstein and Fraiman's. Nevertheless, considering there are only a few experimental measurements of EEY below $1 \mathrm{keV}$ and that these measurements exhibit a large scatter [35], the agreement between the data of Fig. 3 is found to be quite good.

The energy spectrum is an interesting quantity to estimate the consistency of a MC code. For instance, at high energy it gives the contribution of primary (or backscattered) electrons. Most of the backscattered electrons are reflected by the surface after a single elastic interaction and no energy loss. However, the electrons that have been backscattered after experiencing a single inelastic interaction will be emitted out of the target material with a typical energy loss $\hbar \omega$ representative of the interaction. On an energy spectrum, that leads to a typical peak at the characteristic energy $E=E_{0}-\hbar \omega$ with $E_{0}$ the incident electron beam energy. Usually for backscattered electrons, the spectra are given in function of the energy loss $\hbar \omega=E_{0}-E$. The part of the energy loss spectrum corresponding to the backscattered electrons in silicon is reported in Fig. 4.

A relatively good agreement is found between MC simulations and experimental measurements (Fig. 4). The peaks corresponding to the volume plasmon excitation (vp) and its multiples appear clearly at $\hbar \omega=16.8,33.6$ and $50.8 \mathrm{eV}$. The surface plasmon excitation (sp) corresponds to the shoulder at $11.6 \mathrm{eV}$. It is less noticeable than the volume plasmon excitations on both simulated and experimental spectra [36]-[37]. The discrepancies between spectra (height and width of the peaks) may mainly result from the experimental set-up (angle between the electron beam and the sample, angle between the sample and the analyzer) or the surface state (oxidation). 


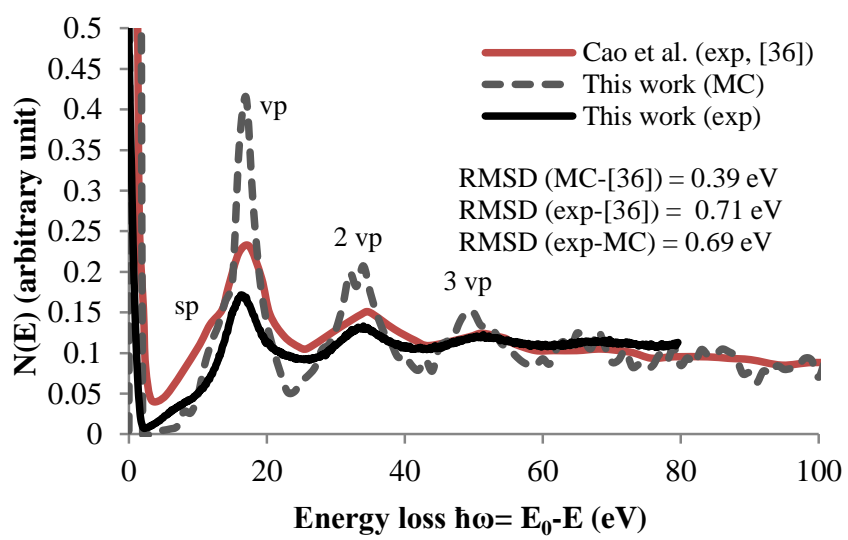

Fig.4. Energy loss spectrum for backscattered electrons in silicon (after [25]). Our experimental spectrum corresponds to $\mathrm{E}_{0}=1990 \mathrm{eV}$ with an accuracy of $0.1 \mathrm{eV}$ whereas our MC spectrum (dashed line with an accuracy of $0.5 \mathrm{eV}$ ) and the experimental measurements of Cao et al. [36] are for $\mathrm{E}_{0}=1000 \mathrm{eV}$. The RMSD calculations are given for the energy loss axis.

At lower energy, the energy spectrum gives the contribution of the secondary electrons from which Auger electrons are easily identified. The Auger electrons are secondary electrons born from collisions with core electrons of the solid. They are emitted out of the solid with a typical energy $E$ that depends on the core levels involved in the interaction. This energy may be described by the formula of Chung and Jenkins [38].

$$
E_{v x y(Z)}=E_{v(Z)}-\frac{1}{2}\left[E_{x(Z+1)}-E_{x(Z)}\right]-\frac{1}{2}\left[E_{y(Z+1)}-E_{y(Z)}\right]
$$

In the formula, an upcoming electron collides with an electron from the v-shell. To fill the newly created vacancy, an electron from the $\mathrm{x}$-shell goes to the $\mathrm{v}$-shell. This electron loses the difference in energy between the $\mathrm{x}$-shell and the $\mathrm{v}$ shell. It transmits this excess of energy to an electron in the yshell that becomes nearly free. Table I reports the energies for the Auger transitions involving the L1-shell and L2,3-shell in silicon along with their probabilities. Fig. 5 shows the MC results for an energy spectrum of Auger electrons in silicon. Our MC simulations are in fairly good agreement with the MC simulations and the experimental results of Cao et al. [36] for the $\mathrm{L}_{2,3} \mathrm{VV}$ and the $\mathrm{L} 1 \mathrm{~L}_{2,3} \mathrm{~V}$ transitions.

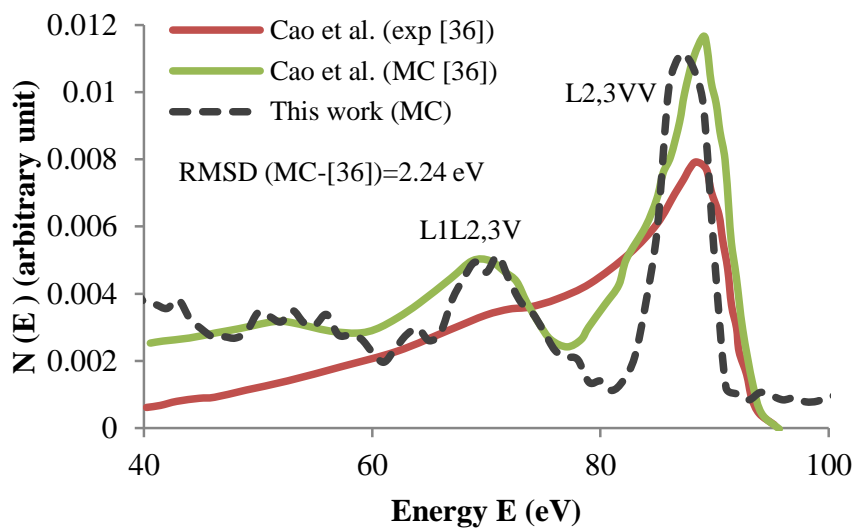

Fig.5. Energy spectrum for the $\mathrm{L}_{2,3} \mathrm{VV}$ and $\mathrm{L}_{2} \mathrm{~L}_{2,3} \mathrm{~V}$ Auger transitions in silicon at $\mathrm{E}_{0}=1000 \mathrm{eV}$ (after [25]). Full lines are the MC simulation and the experimental measurements of [36]. The dashed line is our MC simulation given with an accuracy of $1 \mathrm{eV}$. The RMSD value is given for the energy loss axis.
Table I. Energies for the Auger transitions from the $\mathrm{L}_{2,3}$-shell and the L1-shell in silicon. The probabilities are from the code Geant4 [15], [39].

\begin{tabular}{|c|c|c|c|}
\hline $\begin{array}{l}\text { Auger } \\
\text { Transitions }\end{array}$ & $\begin{array}{l}\text { Value in the } \\
\text { literature in } \\
\mathrm{eV}\end{array}$ & $\begin{array}{l}\mathrm{MC} \text { value in } \\
\mathrm{eV}\end{array}$ & $\begin{array}{l}\text { Probability of } \\
\text { Auger transi- } \\
\text { tion [39] }\end{array}$ \\
\hline $\mathrm{L}_{2,3} \mathrm{VV}$ & $\sim 89[36]$ & $\sim 86$ & 1 \\
\hline $\mathrm{L}_{1} \mathrm{~L}_{2,3} \mathrm{~V}$ & $\sim 70[36]$ & $\sim 68$ & 0.956 \\
\hline $\mathrm{L} 1 \mathrm{VV}$ & & $\begin{array}{c}\sim 134 \text { (hardly } \\
\text { visible) }\end{array}$ & 0.041 \\
\hline
\end{tabular}

\section{IONIZING DOSES}

\section{A. Verification}

The ionizing dose is the deposited energy by unit of mass of a target material subsequent to interactions with the electrons of the solid. The code OSMOSEE enables the calculation of ionizing dose profiles down to a few $\mathrm{eV}$. As depicted in Fig. 6, at $1 \mathrm{keV}$ MC simulated ionizing doses from the code OSMOSEE are in relatively good agreement with those of Akkerman et al. [8], and of Valentin et al. [9]. However, at $100 \mathrm{eV}$ there is a significant discrepancy with the calculations of Valentin et al. who use the Geant4-MicroElec library extension.

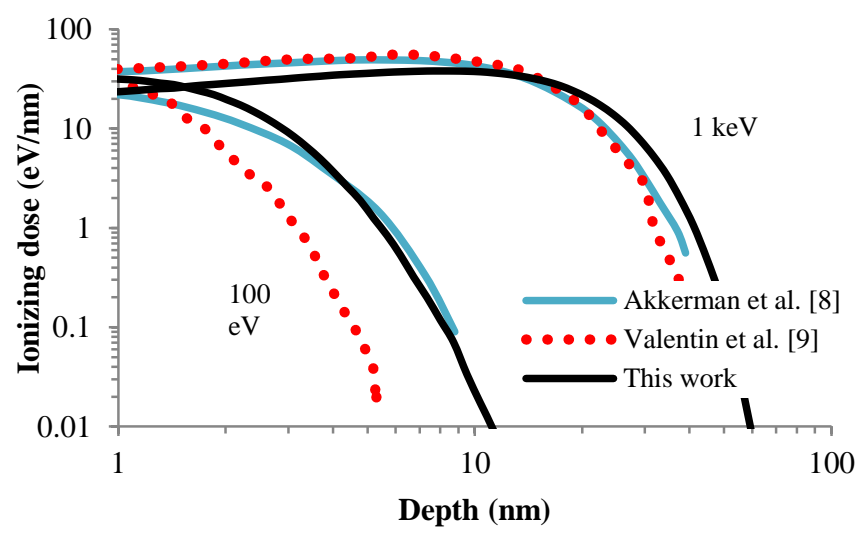

Fig.6. Ionizing dose profiles in eV/nm for electrons of energy $\mathrm{E}_{0}=100 \mathrm{eV}$ and $\mathrm{E}_{0}=1 \mathrm{keV}$ in silicon. The data come from [8]-[9] (after [25]).

The discrepancies between those codes may be mainly explained by two parameters: the energy cutoff at which the electrons come to rest in the solid, and the mean free paths. The effect of the energy cutoff is detailed in section V. C. In their codes, Valentin et al. use an energy cutoff of $16.7 \mathrm{eV}$, and Akkerman et al. of $5 \mathrm{eV}$. In the code OSMOSEE this energy is equivalent to $e \chi=4.05 \mathrm{eV}$ in the vacuum for silicon. Similarly, for aluminum, the energy cutoff is equivalent to $W=4.25 \mathrm{eV}$ in the vacuum. MC simulations strongly depend on the implemented elastic and inelastic mean free paths. The probability of the elastic scattering is depicted in Fig. 7 for the code OSMOSEE, the Geant4-MicroElec model of Valentin et al. [9], and the calculations of Akkerman et al [8]. It presents at low energy a large dispersion from model to model (Fig. 7).

The total number of elastic scatterings along the path of an incident electron will depend on the relative importance of the elastic mean free path compared to the inelastic mean free path. As can be seen on Fig. 7 at $1 \mathrm{keV}$ the probabilities of the 


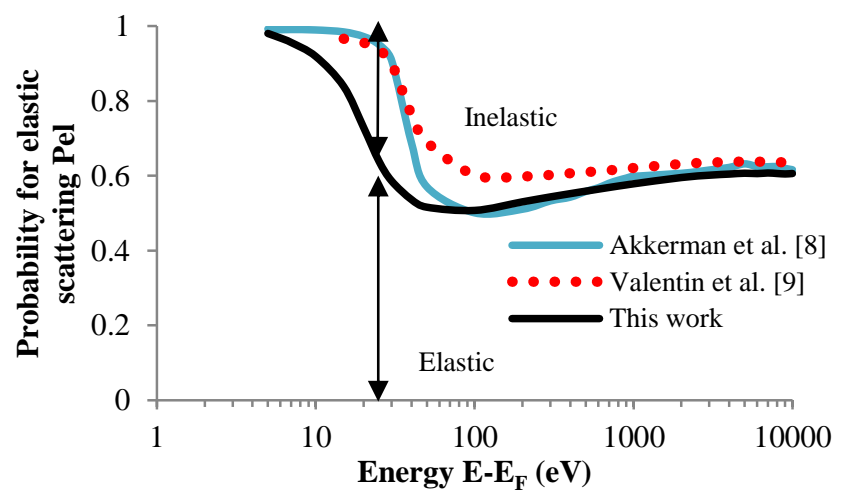

Fig.7. Probability of elastic scattering calculated from the elastic and inelastic mean free paths for electrons in silicon in [8]-[9] and in the code OSMOSEE (after [25]).

elastic interaction given by the three models are close to one another. That leads to dose profiles in relatively good agreement (Fig. 6). On the other hand, as can be seen in Fig. 7, at $100 \mathrm{eV}$, Valentin et al. [9] give a probability of elastic scattering $20 \%$ larger than the two other works that remain close to each other. Accordingly, the dose profile of [9] is relatively different from the two others. In the case of Valentin et al. [9], the electrons undergo a larger number of elastic interactions before being stopped by some inelastic interactions which will produce some important energy losses. The transport of electrons is a kind of random walk motion that limits the penetration of the electrons deeply in the target material. In the case of the two others simulations (code OSMOSEE and [8]), the number of elastic interactions is far lower (Fig. 7). That allows the electrons to go deeper in the material. In comparison with elastic interactions, during inelastic interactions, the electrons are deflected with a small angle, except at very low energy. As a result, the electrons tend to continue their trajectory and may go further in the solid. This trend can be seen on the ionizing dose profiles of Fig. 6. In addition, the three codes employ different elastic cross sections. Down to $\sim 50 \mathrm{eV}$, the code OSMOSEE uses the cross sections from the code ELSEPA of Salvat and co-workers [21]. Similarly, Valentin et al. [9] use cross sections from the ICRU database [40] which is based on the code ELSEPA. However below $50 \mathrm{eV}$, the Mott's model (or partial waves) has been extrapolated down to $10 \mathrm{eV}$ in the code OSMOSEE, whereas Akkerman et al. [8] and Valentin et al. [9] employ the cross sections for $\mathrm{CS}_{2}$ from ab initio calculations by Bettega et al. [41].

\section{B. Ionizing dose profiles}

In this section, we present calculations of ionizing dose profiles for incident electrons of energy between $10 \mathrm{eV}$ and $1 \mathrm{keV}$. In the code OSMOSEE, the ionizing doses are calculated by tracking the secondary electrons until they cannot be emitted out of the solid. The ionizing dose is plotted as a function of the depth of the target material in Fig. 8 for silicon. Fig. 9 presents a comparison with aluminum targets for several energies. Fig. 9 shows that the ionizing dose calculations are very close for silicon and aluminum. For example, at $200 \mathrm{eV}$, the maximum discrepancy is of $15 \%$.

Fig. 8 and Fig. 9 show that from 20 to $200 \mathrm{eV}$, the ionizing dose near the surface increases while the electrons go slightly deeper into the solid. On the contrary, above $200 \mathrm{eV}$, the ionizing dose near the surface decreases while the electrons go strongly deeper into the solid. This behavior must be compared with the practical range [42] of electrons. Fig. 10a shows the practical range of electrons in silicon and Fig. 10b in aluminum.

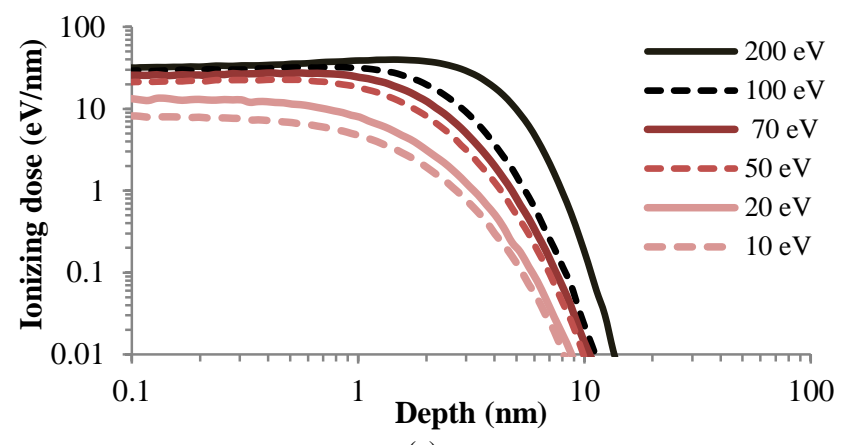

(a)

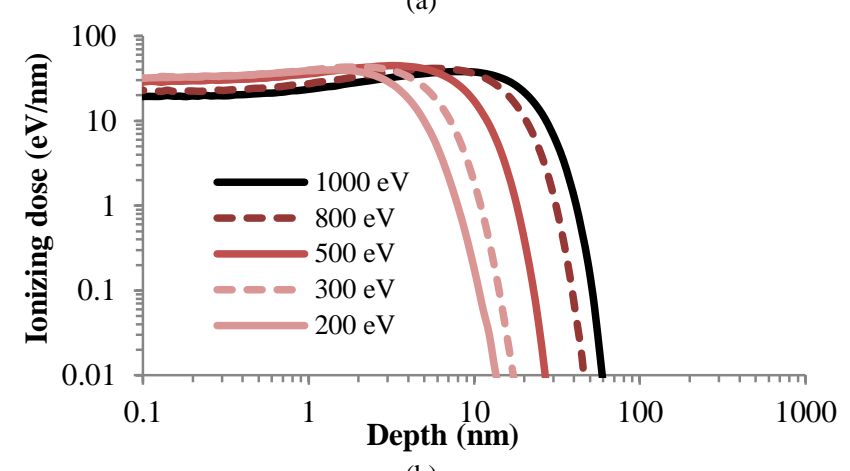

(b)

Fig. 8. Ionizing dose calculations for electrons in silicon from the code OSMOSEE at low energy (a) below $200 \mathrm{eV}$ (b) above $200 \mathrm{eV}$.

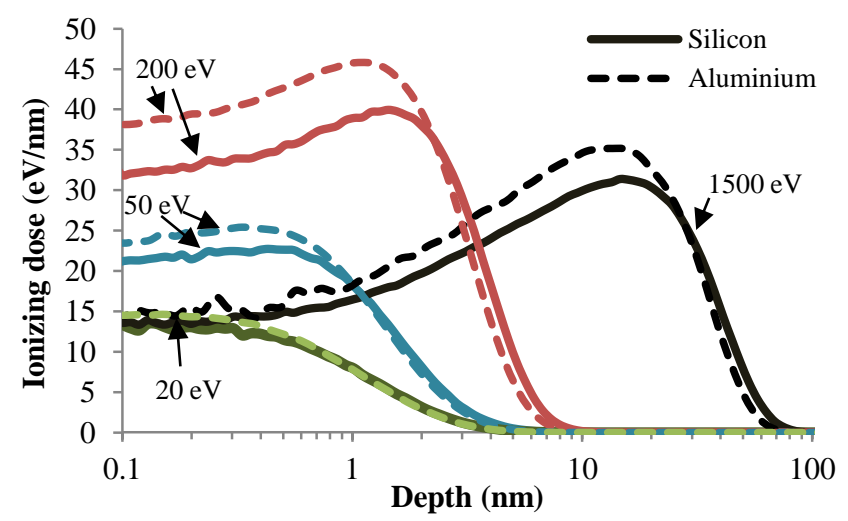

Fig. 9. Comparison of ionizing dose calculations for electrons in aluminum and in silicon from the code OSMOSEE at low energy.

At low energy, below $\sim 100 \mathrm{eV}$, the range of incident electrons remains constant. It does not increase with the incident energy [14], [42] (Fig. 10). At these energies, the transport of electrons is mainly driven by the elastic scattering. Indeed at low energy, the elastic mean free path becomes an order of magnitude lower than the inelastic mean free path. At these energies, the electrons follow a random walk motion until an inelastic interaction forces them to stop in the solid. As a result, the practical range stabilizes and does not decrease anymore with the decreasing energy. Accordingly, between $10 \mathrm{eV}$ up to around $100 \mathrm{eV}$ the range of incident electrons remains constant with a value of a few nm. It shows that, for simula- 
tions of new generation devices of small dimensions, the electron transport may be stopped at a very low energy to be accurate. In this range of energy, the incident electrons deposit their energy near the surface in a volume that remains identical whatever the incident energy is. Accordingly, the ionizing dose increases with the increasing energy. Beyond this energy range, the practical range increases faster, and the ionizing dose is deposited deeper in the material. As a result, the ionizing dose level decreases with the increasing energy.

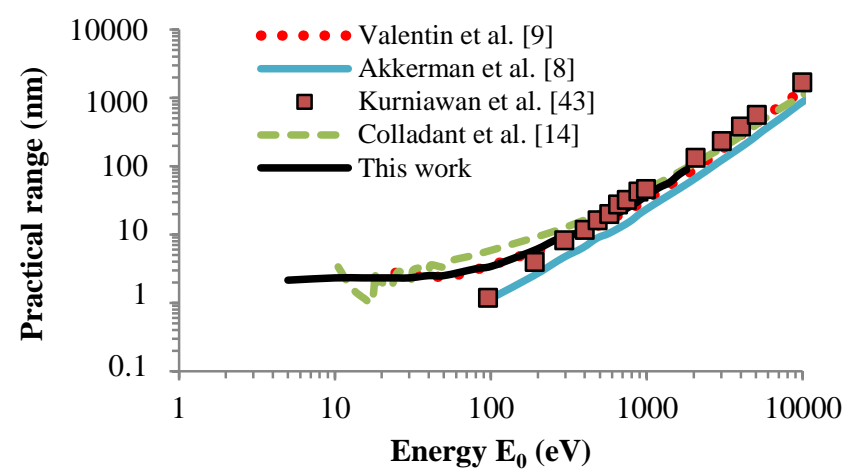

(a)

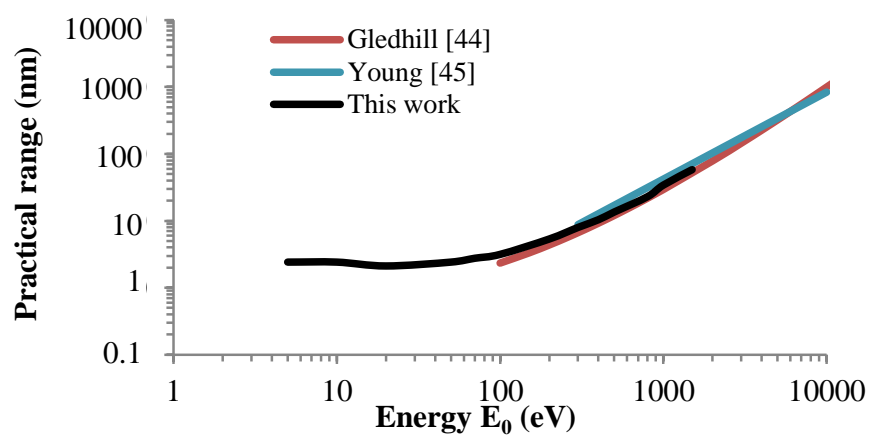

(b)

Fig.10. Practical ranges for electrons (a) in silicon (after [25]) and (b) in aluminum. The data come from [8]-[9], [14], [43]-[45]. The practical range is estimated with 100000 incident electrons which correspond to a statistical uncertainty of $\sim 3 \%$. The depth uncertainty is of $\sim 10 \%$.

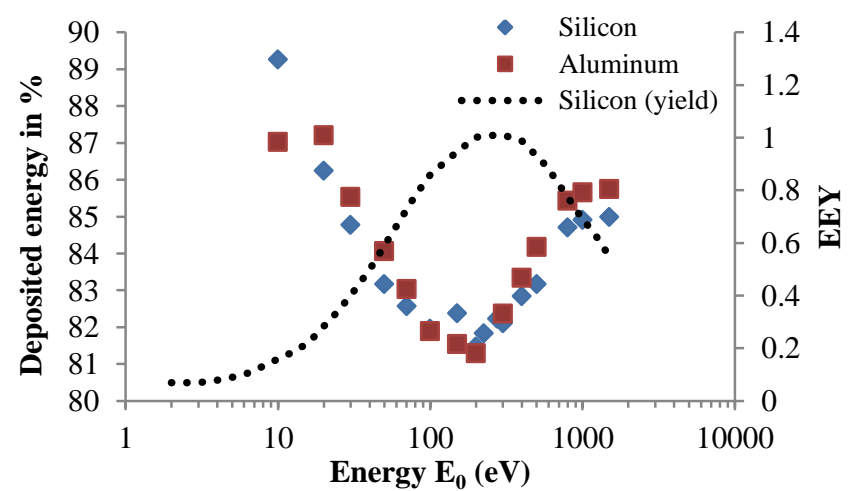

Fig.11. Deposited energy in $\%$ in function of the incident energy for electrons in silicon and in aluminum. This ratio is defined as the deposited energy per electron over the energy of incidence.

This behavior is opposite to the behavior of the EEY. The correlation between the deposited energy and the EEY is obvious in Fig. 11 which represents the fraction of deposited energy in function of the energy of incidence. This ratio in \% is defined as the deposited energy per electron over the energy of incidence. It is interesting to see that the minima for the fraction of the deposited energy are perfectly correlated with the maxima of the EEY for both silicon and aluminum.

Following [8]-[9], the ionizing doses were estimated through a radial distribution at low energy. Fig. 12a to $12 \mathrm{~d}$ show the ionizing dose distributions of electrons as a function of their depth $z$ and their radius $r$ in silicon. The electrons are entering the surface with a normal incidence at the center of

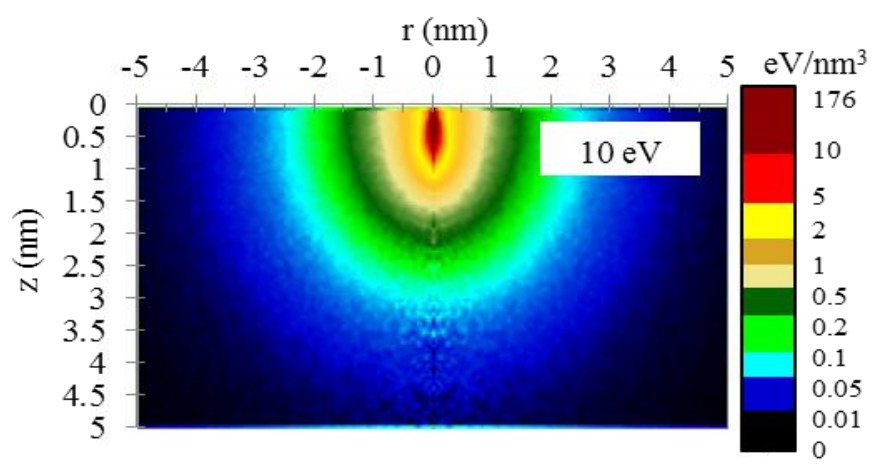

(a)

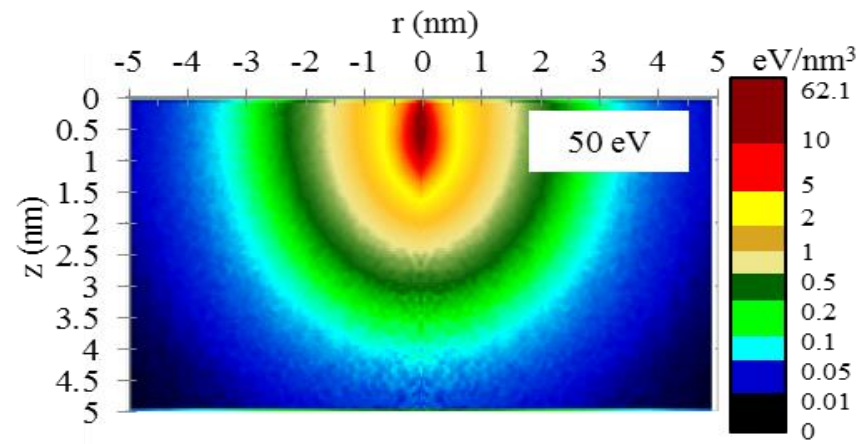

(b)

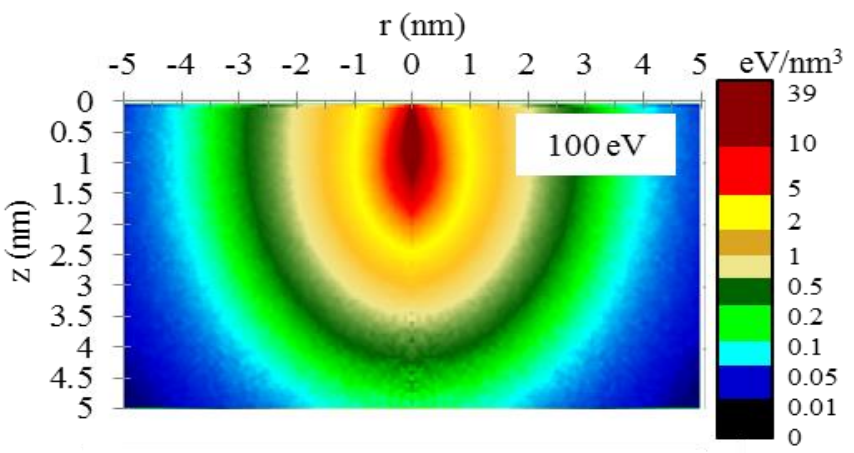

(c)

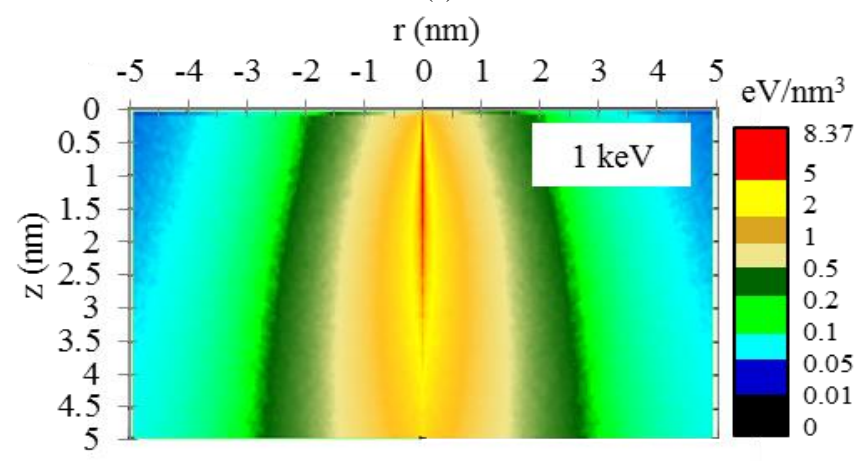

(d)

Fig. 12. Ionizing doses for electrons at low energy in silicon for (a) $10 \mathrm{eV}$ (b) $50 \mathrm{eV}$ (c) $100 \mathrm{eV}$ and (d) $1 \mathrm{keV}$. 
the $\mathrm{z}=0 \mathrm{~nm}$ line. This radial dose profile for electrons represents an average dose level which is not representative of the deposited dose of a single incident electron. It can be seen as a probability for an electron in the track to deposit its energy radially, and may still be useful to calculate the radial extensions of heavy ions tracks that produce a large amount of secondary electrons along their path [5]-[6]. We can see on these figures that below $100 \mathrm{eV}$ the electrons deposit their energy in the first nanometers from the electron beam impact. At higher energy, as they travel deeper into the solid, their deposited energy is spread through a wider volume. As a result, their deposited energy is lower near the surface. The case of silicon is shown in Fig. 12. Similar calculations have been performed for aluminum. The radial dose profile for aluminum is very close to the one of silicon and is not shown here.

\section{Discussion}

At low energy, the accuracy of the spatial extension of the ionizing dose can be discussed. There may be a relatively large difference between the place where a particle loses its energy and where the electron/hole pairs are created by the sub-cascades. Thus, tracking all the secondary electrons is of a great importance for the accuracy of the calculations. For instance, the ionizing dose profiles strongly depend on the energy cutoff at which the secondary electrons are killed. Fig. 13 shows different dose profiles calculated according to different approximations. The full line represents a dose profile when only the tracking of primary electrons is performed. The full tracking of secondary electrons (dashed line on Fig. 13) leads to a significant difference. The ionizing dose is deposited more than twice as deep as in the previous case. And the dose at the surface is reduced by a factor two. When the energy cutoff is increased at $30 \mathrm{eV}$, the production of secondary electrons is limited and the ionizing dose profile tends to the case where only primary electrons are tracked (dotted line vs. full line in Fig. 13).

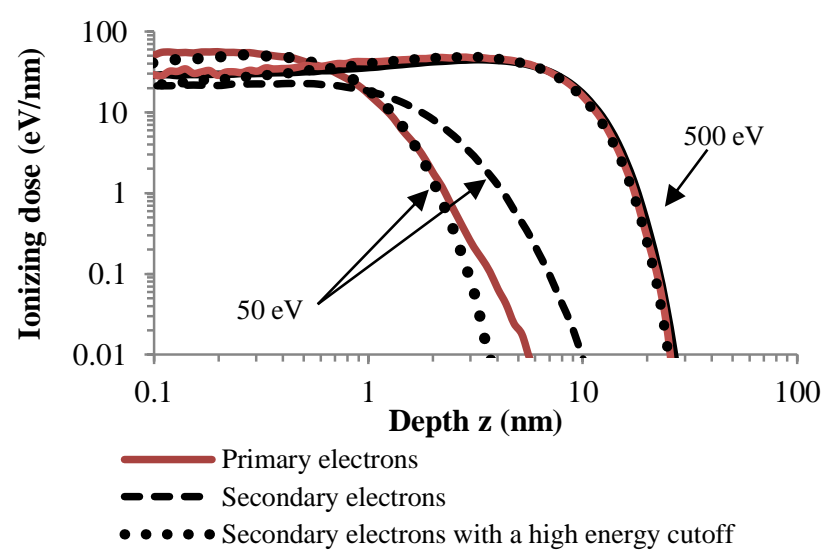

Fig. 13. Ionizing dose calculations by tracking primary electrons (full lines) and secondary electrons (dashed lines). The dotted lines represent the tracking of secondary electrons with an energy cutoff of $30 \mathrm{eV}$ in the solid. At $\mathrm{E}_{0}=500 \mathrm{eV}$, the three curves overlap.

At high energy, the use of a higher energy cutoff does not change significantly the ionizing dose profile. An example is given for $\mathrm{E}_{0}=500 \mathrm{eV}$ on Fig. 13. The extension of the dose due to the secondary electrons is of a few nanometers. At high energy, it becomes negligible compared to the range of the primary electrons that is superior to $10 \mathrm{~nm}$.

Besides, the ionizing dose directly depends on the secondary emission yield: the total deposited energy is the difference between the energy brought to the material by the incident beam reduced to the amount of energy that escapes by means of secondary electron emission. Since the backscattering of electron is mainly driven by the elastic scattering, at low energy the ionizing dose will depend closely on the accuracy of this scattering process.

\section{CONCLUSION}

The transport of electrons in silicon and in aluminum is investigated with a Monte Carlo code down to $10 \mathrm{eV}$. The MC code is based on the complex dielectric function theory and takes into account surface plasmon excitations, phonons, and the surface potential barrier. Simulations from the code are compared with experimental measurements of EEY and energy loss spectra performed in ultrahigh vacuum on Ar-etched samples. The EEY is given below $2 \mathrm{keV}$ and down to $20 \mathrm{eV}$. Simulation results are in good agreement with experimental data. For the EEY, the discrepancy is of less than $19 \%$ between our experimental measurements and our simulations above $50 \mathrm{eV}$ for aluminum and silicon. Our data performed on bulk solids are also consistent with Bronstein and Fraiman's measurements on ultrahigh evaporated samples. For the energy loss spectra of silicon, the peaks corresponding to the Auger electrons, to the volume plasmon excitations and its multiples are well defined. These results enable the calculations of practical ranges and ionizing doses down to $10 \mathrm{eV}$ for aluminum and silicon. At low energy, below $100 \mathrm{eV}$, the practical range is demonstrated to reach a plateau region and stabilizes to some few nanometers for both silicon and aluminum. Accurate range/energy functions are of a great interest in the formulation of new accurate secondary emission model [42]. The ionizing dose profiles that have been calculated for energies down to $10 \mathrm{eV}$ are demonstrated to be closely related to the secondary electron emission yield. The ionizing dose depth profiles commonly calculated at higher energies neglect the effect of the secondary electron emission yield, however, the deposited energy is reduced by the amount of electrons that get out from the irradiated material. The deposited ionizing dose is negatively correlated with the EEY. It is reduced by nearly $10 \%$ when the EEY reaches a value of 1 . The effect will be larger for materials with high secondary emission capacity that can overpass a value of 2 .

\section{REFERENCES}

[1] R. Cimino, L. A. Gonzalez, R. Larciprete, A. Di Gaspare, G. Iadarola, and G. Rumolo, "Detailed investigation of the low energy secondary electron yield of technical $\mathrm{Cu}$ and its relevance for the LHC", Phys. Rev. ST Accel. Beams, vol. 18, no. 5, p. 51002, May 2015.

[2] R. Udiljak, D. Anderson, M. Lisak, J. Puech, and V. E. Semenov, "Multipactor in waveguide iris", IEEE Trans. Plasma Sci., vol. 35, no. 2, pp. 388-395, Apr. 2007.

[3] H. Seiler, "Secondary electron emission in the scanning electron microscope", J. Appl. Phys., vol. 54, no. 11, pp. R1-R18, Nov. 1983. 
[4] G. Larrieu, and X. L. Han, "Vertical nanowire array-based field effect transistors for ultimate scaling", Nanoscale, vol. 5, no. 6, pp. 24372441, Mar. 2013

[5] M. P. King, R. A. Reed, R. A. Weller, M. H. Mendenhall, R. D. Schrimpf, N. D. Pate, E. A. Auden, and S. L. Weeden-Wright, "Radial characteristics of heavy-ion track structure and implications of deltaray events for microelectronics", Appl. Phys. Lett., vol. 101, no. 5, p. 53509, Jul. 2012.

[6] C. Inguimbert, R. Ecoffet, and D. Falguère, "Electron Induced SEUs: Microdosimetry in Nanometric Volumes", IEEE Trans. Nucl. Sci., vol. 62 , no. 6, pp. 2846-2852, Dec. 2015.

[7] A. Akkerman, T. Boutboul, A. Breskin, R. Chechik, A. Gibrekhterman, and Y. Lifshitz, "Inelastic electron interactions in the energy range $50 \mathrm{eV}$ to $10 \mathrm{keV}$ in insulators: Alkali halides and metal oxides", Phys. Stat. Sol (b), vol. 198, no. 2, pp. 769-784, Dec. 1996.

[8] A. Akkerman, M. Murat, and J. Barak, "Monte Carlo calculations of electron transport in silicon and related effects for energies of 0.02200 keV", J. Appl. Phys., vol. 106, no. 11, p. 113703, Dec. 2009

[9] A. Valentin, M. Raine, J.-E. Sauvestre, M. Gaillardin, and P. Paillet, "Geant4 physics processes for microdosimetry simulation: Very low energy electromagnetic models for electrons in silicon", Nucl. Instrum. Methods Phys. Res. B., vol. 288, pp. 66-73, Oct. 2012.

[10] M. Raine, A. Valentin, M. Gaillardin, and P. Paillet, "Improved simulation of ion track structures using new Geant 4 models - Impact on the modeling of advanced technologies response", IEEE Trans. Nucl. Sci., vol. 59 , no. 6 , pp. 2697-2703, Dec. 2012.

[11] M. Raine, M. Gaillardin, and P. Paillet, "Geant4 physics processes for silicon microdosimetry simulation: Improvements and extension of the energy-range validity up to $10 \mathrm{GeV} /$ nucleon”, Nucl. Instrum. Methods Phys. Res. B, vol. 325, pp. 97-100, Apr. 2014.

[12] H.-J. Fitting, E. Schreiber, J.-Ch. Kuhr, and A. Von Czarnowski, "Attenuation and escape depths of low-energy electron emission", $J$. Electron Spectrosc. Relat. Phenom., vol. 119, no. 1, pp. 35-47, Jul. 2001.

[13] J.-Ch. Kuhr and H.-J. Fitting, "Monte Carlo simulation of electron emission from solids", J. Electron Spectrosc. Relat. Phenom., vol. 105, no. 2-3, pp. 257-273, Dec. 1999.

[14] T. Colladant, A. L'Hoir, J.E. Sauvestre, and O. Flament, "Monte-Carlo simulations of ion track in silicon and influence of its spatial distribution on single event effects", Nucl. Instrum. Methods Phys. Res. B, vol. 245, no. 2, pp. 464-474, Apr. 2006.

[15] GEANT4: A toolkit for the simulation of particles through matter. May 2014 [Online]. Available: http://geant4.web.cern.ch/geant4/

[16] J. Roupie, "Contribution à l'étude de l'émission électronique sous impact d'électrons de basse énergie $(<=1 \mathrm{keV})$ : application à l'aluminium (in French)", Ph. D. dissertation, ISAE, Toulouse, France, 2013.

[17] J. Roupie, O. Jbara, T. Tondu, M. Belhaj, and J. Puech, "The study of electron emission from aluminum in the very low primary energy range using Monte Carlo simulations", J. Phys. D. Appl. Phys., vol. 46, no. 12 , p. 125306 , Feb. 2013.

[18] C. Inguimbert, M. Belhaj, J. Puech, and J. Pierron, "Monte Carlo simulation of the electron range/energy relationship in the sub-keV energy domain", presented at the $8^{\text {th }}$ Int. MULCOMPIM Workshop, Valencia, Spain, Sep. 17-19, 2014. [Online]. Available: DOI 10.13140/2.1.2159.4880

[19] T. Gineste, M. Belhaj, G. Teysedre, and J. Puech, "Investigation of the electron emission properties of silver: from exposed to ambient atmosphere Ag surface to ion-cleaned Ag surface”, Appl. Surf. Sci., vol. 359, pp. 398-404, Dec. 2015.

[20] T. Gineste, "Emission électronique sous impact d'électrons : applications spatiales (in French)", Ph. D. dissertation, ISAE, Toulouse, France, 2015.

[21] F. Salvat, A. Jablonski, and C. J. Powell. "ELSEPA - Dirac partialwave calculation of elastic scattering of electrons and positrons by atoms, positive ions and molecules", Comput. Phys. Commun., vol. 165, no. 2, pp. 157-190, Jan. 2005.

[22] R. H. Ritchie, and A. Howie, "Electron excitation and the optical potential in electron microscopy", Philos. Mag. vol. 36, no. 2, pp. 463481, Aug. 1977.

[23] Handbook of Optical Constants of Solids, 1st ed., edited by E. D. Palik, Academic Press, Burlington, 1997.

[24] J. P. Ganachaud, and M. Cailler, "A Monte-Carlo calculation of the secondary electron emission of normal metals: I. the model", Surf. Sci., vol. 83, no. 2, pp. 498-518, May 1979.
[25] J. Pierron, C. Inguimbert, M. Belhaj, M. Raine, and J. Puech, "Monte Carlo simulations of low energy electrons in silicon", in Proc. RADECS 2016.

[26] H. Shinotsuka, S. Tanuma, C. J. Powell, and D. R. Penn, "Calculations of electron inelastic mean free paths. X. Data for 41 elemental solids over the $50 \mathrm{eV}$ to $200 \mathrm{keV}$ range with the relativistic full Penn algorithm", Surf. Interface. Anal., vol. 47, no. 9, pp. 871-888, Jul. 2015.

[27] C.J. Tung, J.C. Ashley, and R.H. Ritchie, "Electron inelastic mean free paths and energy losses in solids II: Electron gas statistical model", Surf. Sci, vol. 81, no. 2, pp. 427-439, Mar.1979.

[28] M. Gryzinski, "Classical theory of atomic collisions. I. Theory of inelastic collisions", Phys. Rev. Lett., vol. 138, no. 2A, pp. A336-A358, Apr. 1965.

[29] X. Llovet, C. Powell, F. Salvat, and A. Jablonski, "Cross sections for inner-shell ionization by electron impact", J. Phys. Chem. Ref. Data., vol. 43, no. 1, p. 13102, Mar. 2014.

[30] J. Fernandez-Varea, F. Salvat, and M. Dingfelder, "A relativistic optical-data model for inelastic scattering of electrons and positrons in condensed matter", Nucl. Instrum. Methods Phys. Res. B, vol. 229, no. 2, pp. 187-218, Mar. 2005.

[31] J.P. Wang, C. J. Tung, Y.F. Chen, and C. M. Kwei, "The surface effect on $\mathrm{Au} 4 \mathrm{f} \mathrm{X}$-ray photoelectron spectra", Nucl. Instrum. Methods Phys. Res. B, vol. 108, no. 3, pp. 331-338, Feb. 1996.

[32] A. Akkerman, and M. Murat, "Electron-phonon interactions in silicon: Mean free paths, related distributions and transport characteristics", Nucl. Instrum. Methods Phys. Res. B, vol. 350, pp. 49-54, May 2015.

[33] R. D. Narayan, R. Miranda, and P. Rez, "Monte Carlo simulation for the electron cascade due to gamma rays in semiconductor radiation detectors", J. Appl. Phys., vol. 111, no. 6, p. 64910, Mar. 2012.

[34] M. Bronstein, and B. S. Fraiman, Vtorichnaya Elektronnaya Emissiya (in Russian), Nauka, Moscow, 1969.

[35] D. C. Joy, "A database on electron-solid interactions", Scanning, vol. 17, no. 5, pp. 270-275, Dec. 2006.

[36] N. Cao, B. Da, Y. Ming, S. F. Mao, K. Goto, and Z. J. Ding, "Monte Carlo simulation of full energy spectrum of electrons emitted from silicon in Auger electron spectroscopy", Surf. Interface. Anal., vol. 47, no. 1, pp. 113-119, Oct. 2014.

[37] M. Dapor, L. Calliari, and G. Scarduelli, "Comparison between Monte Carlo and experimental aluminum and silicon energy loss spectra", Nucl. Instrum. Methods Phys. Res. B, vol. 269, no. 14, pp. 1675-1678, Jul. 2011.

[38] M. F. Chung, and L. H. Jenkins, “ Auger electron energies of the outer shell electrons", Surf. Sci., vol. 22, no. 2, pp. 479-485, Sep. 1970.

[39] S. T. Perkins, M. H. Chen, D. E. Cullen, and J. H. Hubbell, "Tables and Graphs of Atomic Subshell and Relaxation Data Derived from the LLNL Evaluated Atomic Data Library (EADL), Z=1-100", Lawrence Livermore Nat. Lab., Livermore, USA, Rep. UCRL-50400-V-30, 1991.

[40] ICRU, "Elastic scattering of electrons and positrons", Journal of the ICRU, Oxford: Oxford University Press, Rep. 77, vol. 7, no. 1, pp. 1162, Jun. 2007.

[41] M. H. Bettega, M. A. P. Lima, and L. G. Ferreira, "Electron collisions with CS2", J. Phys. B. At. Mol. Opt. Phys., vol. 38, no. 13, pp. 20872095, Jun. 2005

[42] C. Inguimbert, J.Pierron, M. Belhaj, and J. Puech, "Extrapolated range expression for electrons down to $\sim 10 \mathrm{eV}$ ", presented at the IEEE NSREC Conf., Portland, USA, Jul. 11-15, 2016. [Online]. Available: https://hal.archives-ouvertes.fr/hal-01372004

[43] O. Kurniawan, and V.K.S. Ong, "Investigation of range-energy relationships for low-energy electron beams in silicon and gallium nitride", Scanning, vol. 29, no. 6, pp. 280-286, Nov. 2007.

[44] J. A. Gledhill, "The range-energy relation for 0.1-600 keV electrons", $J$. Phys. A; Math., Nucl. Gen., vol. 6, no. 9, pp. 1420-1428, Sep. 1973.

[45] J. R. Young, "Penetration of electrons and ions in aluminum", J. Appl. Phys., Vol. 27, no. 1, pp. 1-4, Jan. 1956. 